\title{
Influence of Ti content on the Structural and Magnetic Properties of
}

\author{
$\mathrm{Li}_{1-\mathrm{x}} \mathrm{Ti}_{\mathrm{x}} \mathrm{La}_{\mathrm{y}} \mathrm{Fe}_{2-\mathrm{y}} \mathrm{O}_{4}$ Ferrite; $\mathbf{0 . 1} \leq \mathbf{x} \leq \mathbf{0 . 9}$ and $\mathbf{y}=\mathbf{0 . 1}$.
}

\author{
E.H. Elkhawas* \\ Department of Basic Science, Higher Technological Institute, Tenth of \\ Ramadan City, Egypt. \\ E.Mail:enash12@hotmail.com
}

The variation of the transition phenomena in the spinel structure; of $\mathrm{Li}_{1-x} \mathrm{Ti}_{x} \mathrm{La}_{y} \mathrm{Fe}_{2-y_{-}} \mathrm{O}_{4}$ ferrite from the ferrimagnetic to paramagnetic state was observed by substituting Ti for $\mathrm{Li}$ in compound. XRD analysis was carried out in order to find a correlation between the average lattice parameter, crystal size on Ti content. Drastic change was observed between $x=0.5$ and $x=0.7$ in the magnetic measurements which agrees well with the percolation theory. The magnetization as calculated from susceptibility measurements as a function of temperature and magnetic fields was interpreted in view of Ti contents. The measurements showed that the magnetization increases with increasing magnetic field which is a normal trend of ferrites in the ferrimagnetic region. The susceptibility decreases as the temperature increases due to the increase of the lattice vibration as well as the spin disorder. In addition, the existence of small quantity of La ions influences the magnetic properties of such materials. The hysteresis loops of the investigated samples showed that by increasing $\mathrm{Ti}$ and decreasing Li contents the magnetic parameters $M_{s}$ and $M_{r}$ decrease as $H_{c}$ increases. The calculated effective magnetic moment $\mu_{\text {eff }}$ also varied with $\mathrm{Ti}$ content, taking into consideration the screening effect of both $\mathrm{Ti}$, $\mathrm{Li}$ and $\mathrm{La}^{3+}$ ion.

\section{Introduction:}

Ferrites are of great importance from the point of view of scientific and industrial applications [1]. They have many applications in a great variety of technical fields such as electric chokes, cores for AF and RF transformers, magnetic recording media, magneto-optical readers, rod antennas, recently radar absorbing materials (RAM). The RAM has the property to change the electromagnetic radiation energy to thermal energy. This property is used in telecommunication, in cellular phones and reception/transmission antennas [2-3]. Some composites of Ni-Zn ferrite with polymer can be used as high performance RAM with a microwave absorbtion up to $96.9 \%(\approx 15 \mathrm{~dB})$. The 
higher electrical and good magnetic properties of such composites make them more applicable materials. Among the most remarkable properties of Li-Ti ferrites there are potential microwave applications according to the narrow ferrimagnetic resonance line width as well as the low values of the loss tangent. Both aspects seem closely related to the magnetic aftereffect phenomena occurring in these ferrites, which can be markedly reduced by appropriate cation substitutions [4-9]

Li-Ti-La ferrite belongs to the ferrimagnetic materials which have a structure of orthoferrites consists of a large cations $\mathrm{La}^{3+}$ and a simple cubic structure with the oxygen anions in the face-centre positions and with the smaller cation, lithium, in the body centre [10]. The metallic ions occupying the tetrahedral (A) and the octahedral (B)sites, which interact with each other through the exchange interaction. Magnetic disorder can be easily introduced into the system by either selective sub lattice dilution or cation distribution.

The reduction of porosity in $\mathrm{Li}-\mathrm{Ti}$ ferrite is due to that, the lithium volatility and oxygen deficiency imply a limitation on the sintering temperature. By introducing a foreign ions (La), an important modification of both structure and magnetic properties can be obtained. [11-14].

By substituting nonmagnetic ions such as Ti for Li ferrite, a very large influence on several of its magnetic properties such as magnetic susceptibility, Curie temperature, etc take place. Ti-ions also have considerable effect on other properties of lithium ferrite like B-site ordering, dielectric behavior, etc [15].

The aim of the present work is to follow up the variation of $\mathrm{T}_{\mathrm{N}}$ (Neel's temperature) and crystallite size with the replacement of $\mathrm{Ti}^{4+}$ ions on the expence of $\mathrm{Li}^{2+}$ ions on the physical properties.

\section{Material and Methods:}

It is well known that the physical properties of ferrite materials depend on the preparation conditions such as sintering time, sintering temperature, heating and cooling rate. So, we have to control and distinguish the preparation conditions for the desired sample. From this point of view, pure analar oxides ( $\mathrm{BDH})$ of $\left(\mathrm{LiOH}, \mathrm{Ti}_{2} \mathrm{O}_{3}, \mathrm{La}_{2} \mathrm{O}_{3}\right.$ and $\left.\mathrm{Fe}_{2} \mathrm{O}_{3}\right)$ is mixed in stoichiometric ratio. The mixed oxides are grinded manually using agate mortar for two hours and pressed into pellets form using uniaxial hydraulic press of pressure $5 \times 10^{8} \mathrm{~N} / \mathrm{m}^{2}$ at room temperature. The obtained pellets are pre-sintered at $\left(800^{\circ} \mathrm{C}\right)$ for 8 hours using heating rate $4^{\circ} \mathrm{C} / \mathrm{min}$. The samples are lifted to cool to room temperature in open atmosphere with the same rate as that of heating. Regrinding were 
carried out for half an hour then pressed again into pellets and finally sintered at $\left(1100^{\circ} \mathrm{C}\right)$ for 16 hours with the same rate as that of pre-sintering.

The obtained pellets were grinded, part of them is used to carry out the Xray diffraction using scintag (USA) X-ray diffractometer dians corporation with target $\mathrm{CuK} \alpha\left(\lambda=1.5418^{\circ} \mathrm{A}(\mathrm{XDR})\right)$ to examine the complete reaction and the formation of single phase cubic structure. The second part of the powder is used to carry out the hysteresis analysis and magnetic susceptibility measurements using Faraday's method from room temperature up to $970 \mathrm{~K}$ at three different magnetic field intensities ranged from 1010 Oe up to 2320 Oe ; the effective magnetic moment is calculated from the reciprocal of the magnetic susceptibility and absolute temperature above the transition temperature about $800 \mathrm{~K}$. The hysteresis was made up using vibrating sample magnetometer (VSM; 9600-1LDJ, USA) at a maximum field of $5 \mathrm{kOe}$.

\section{Results and Discussion:}

Figure (1:a-e) : shows the X-ray diffraction patterns for the ferrite of the general formula $\mathrm{Li}_{1-\mathrm{x}} \mathrm{Ti}_{\mathrm{x}} \mathrm{La}_{\mathrm{y}} \mathrm{Fe}_{2-\mathrm{y}} \mathrm{O}_{4}, 0.1 \leq \mathrm{x} \leq 0.9$ and $\mathrm{y}=0.1$. The pattern shows that all samples exhibit spinel cubic structure as compared with ICDD cards. Moreover, the cation distribution between tetrahedral and octahedral sites can take place according to D.Ravinder [16] and $\mathrm{Xu}$ Yunhui[17]. The estimated lattice parameters of prepared samples are in fair agreement with the published data[18]. Inspection of XRD patterns shows that the intensity ratio differs from that given in XRD cards. This is most likely due to preferred orientation.

Figure (2:a) shows the dependence of Nee'l temperature $\left(\mathrm{T}_{\mathrm{N}}\right)$ on $\mathrm{Ti}$ content, the obtained data shows a decrease in $\mathrm{T}_{\mathrm{N}}$ with Ti-content which may be due to the decrease of the ferrimagnetic region as a result of the masking effect of $\mathrm{Ti}^{3+}$ ions.

No trend is observed for the lattice parameter a, which may be due to rearrangement of cations in A and B sites. This may be explained on the basis of octahedron shrinking [18-20]. This will result in a very small decrease in a lattice parameter with increasing concentration above $\mathrm{x}=0.5$.

Figure $(3: a, b)$ shows representative of magnetization $(M)$ as a function of temperature at different magnetic fields of intensities (1010, 1340, $1660 \mathrm{Oe})$. The data in the figure are drawn for ferrimagnetic region at which the magnetic dipoles are aligned along the field direction. As it is clear Fig.(3:a) the alignment increases with increasing the magnetic field intensity such that the magnetization at $1660 \mathrm{Oe}$ is greater than those at the other two fields. The 
saturation magnetization takes place when all the dipoles are aligned in the field direction [3].
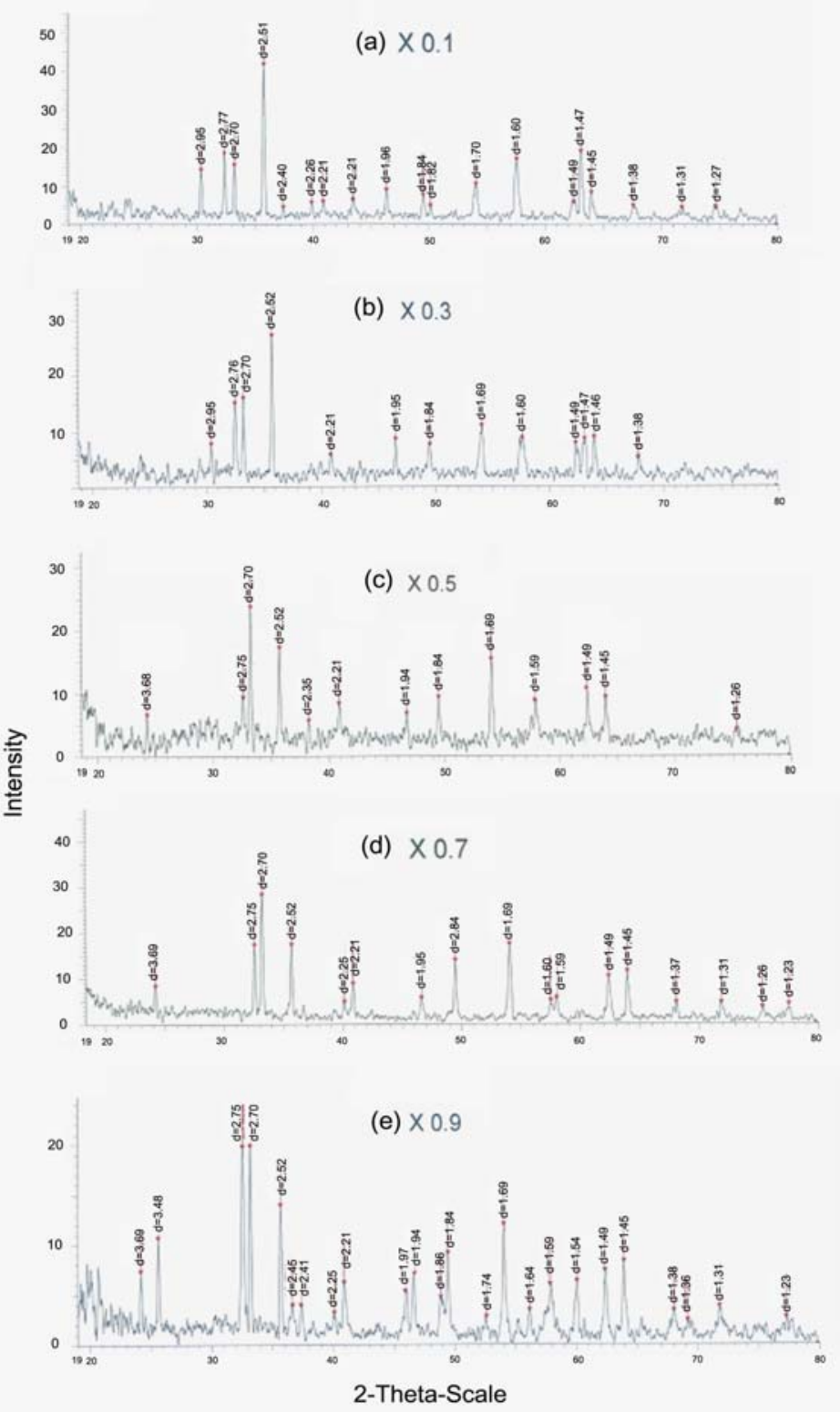

Fig . ( 1:a-e ) X-Ray diffraction patterns for the ferrites $\mathrm{Li}_{1-x} \mathrm{Ti}_{x} \mathrm{La}_{y} \mathrm{Fe}_{2-y} \mathrm{O}_{4}$ $x=0.1,0.3,0.5,0.7,0.9$ and $y=0.1$ 

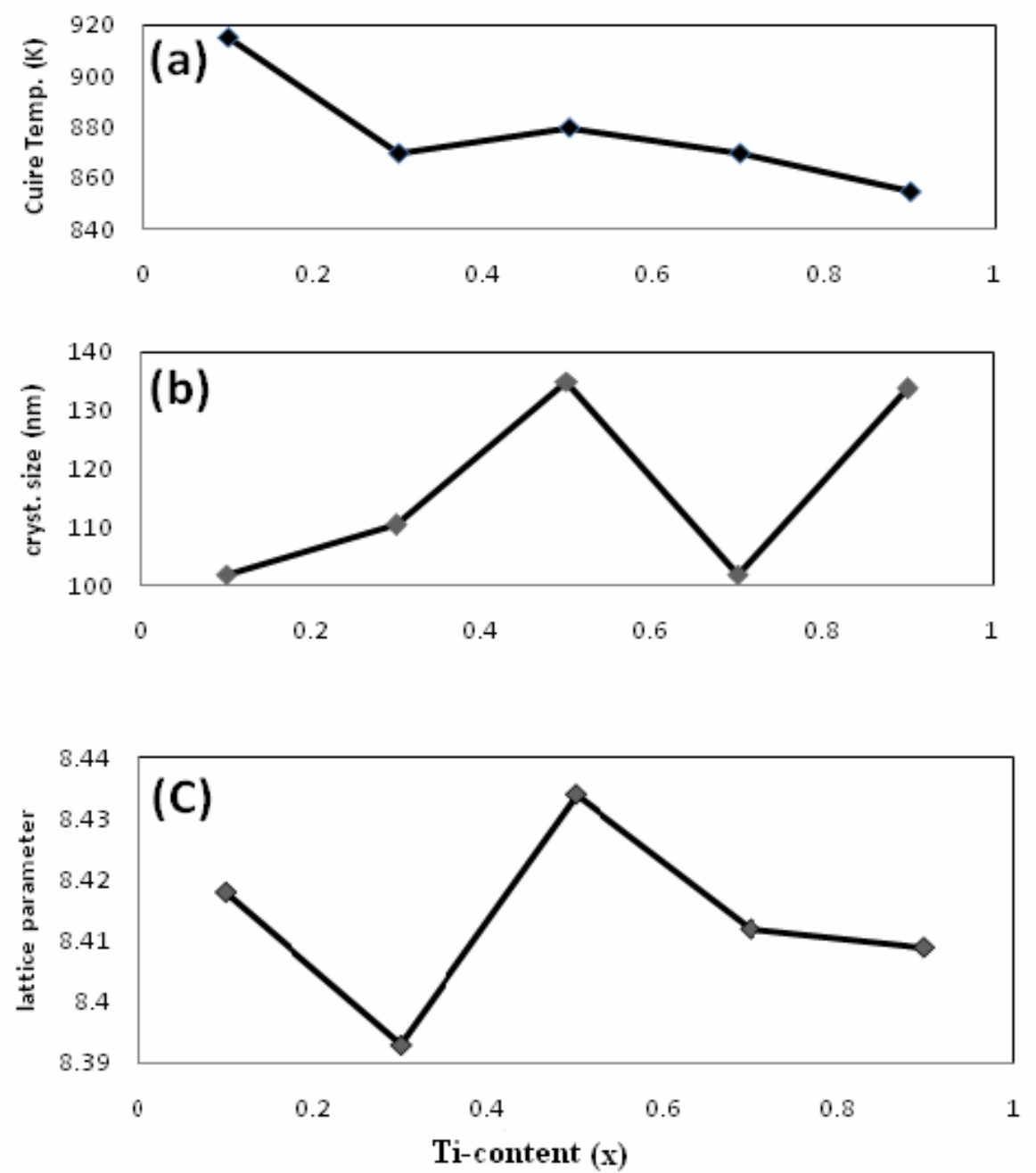

Fig.(2:a-c): Variation of(a) Curie temperature, (b)crystalline size, and (c) Latticeparameter vs. Ti content.

Figure (3: a,b andc) shows the dependence of magnetization on both absolute temperature for different Ti-content. It is obvious that by increasing Ticontent the magnetization gives lowest values which is acceptable result. This is because $\mathrm{Ti}^{3+}$ ions mask the magnetic interaction between $\mathrm{A}$ and $\mathrm{B}$ sublattices, $\mathrm{M}_{\mathrm{AB}}=\mathrm{M}_{\mathrm{B}}-\mathrm{M}_{\mathrm{A}}$. In other words, we expect that some of $\mathrm{Fe}^{3+}$ is transformed into $\mathrm{Fe}^{2+}$ and varying the ratio $\mathrm{Fe}^{2+} / \mathrm{Fe}^{3+}$ [3]. This is confirmed by Fig. (3: d) where the general trend of the data is the decrease in the effective magnetic moment $\mu_{\text {eff. }}$ in which it is calculated from the relation $\mu_{\text {eff. }}=2.83 \sqrt{ } \mathrm{C}$ [19], where $\mathrm{C}$ is the Curie constant (in the region above $\mathrm{T}_{\mathrm{N}}$ ) which is calculated from the data in which the reciprocal of the slope between $\chi^{-1}{ }_{\mathrm{M}}$ versus $\mathrm{T}(\mathrm{K})$ are taken. 
The effect of temperature on magnetization is not pronounced specially for $\mathrm{x}=0.5$. The sample under investigation appears very small change in the first temperature region up to $500 \mathrm{~K}$ after which a remarkable decrease is observed but the effect of the magnetic field was appeared around the Nee'l temperature which makes a splitting between the two regions in the curve Fig.(4). Accordingly, the thermal energy given to the sample is not quite sufficient to free the localized magnetic dipoles [20]. When comparing Fig. (2: a) with Fig. (3: d) one can find that a peculiar behavior exists at $\mathrm{x}=0.5$ which is considered as the critical concentration. This enhances the suggested approach concerning the critical behavior at this concentration.
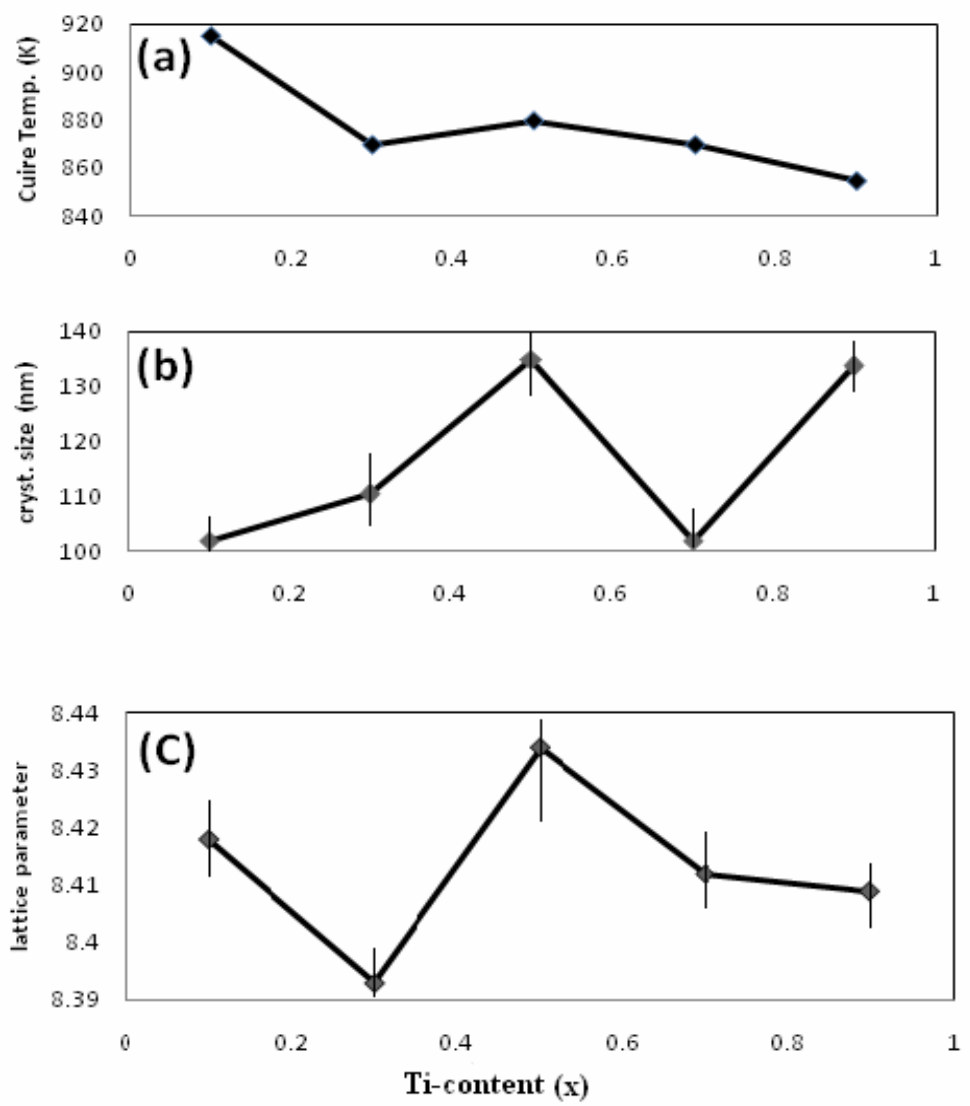

Fig.(2:a-c): Variation of(a) Curie temperature, (b)crystalline size, and (c) Lattice parameter vs. Ti content. 

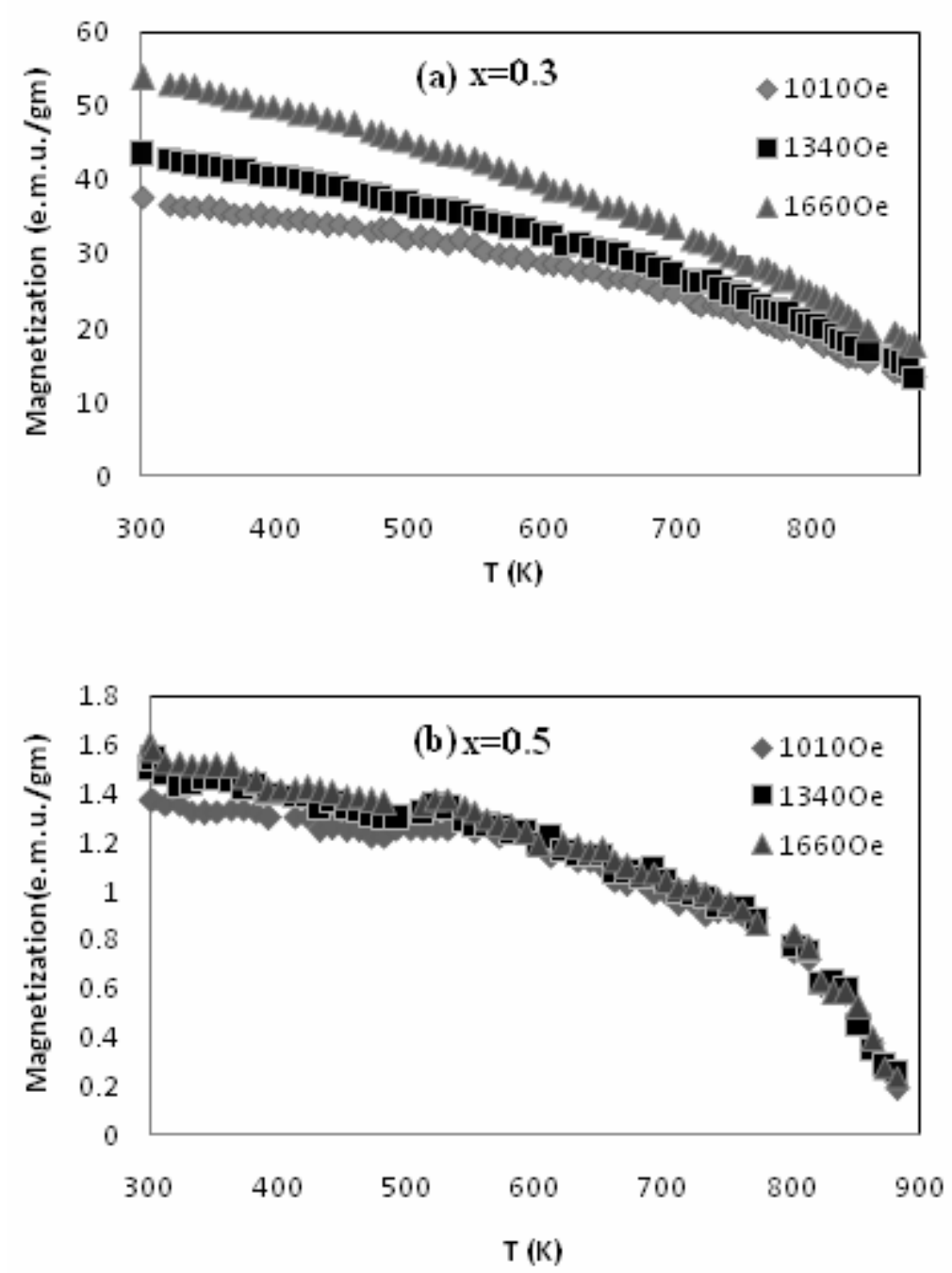

Fig. (3: a, b): Variation of magnetization with temperature $a) x=0.3, b) x=0.5$, at different field strengths.

The values of the Curie-Weiss temperature $(\Theta)$ and the effective magnetic moment which were calculated (above $T_{N}$ ) from the slope of the reciprocal susceptibility of all concentration and at different field strength are reported in Table (1). The data shows the normal behavior of ferrimagnetic material from $\mathrm{x}=0.1$ up to $\mathrm{x}=0.5$ after which an anomalous behavior appeares at $\mathrm{x}=0.7$ and $\mathrm{x}=0.9$. This can be attributed to the rearrangement of $\mathrm{Fe}^{2+}$ and $\mathrm{Fe}^{3+}$ between $\mathrm{A}$ and $\mathrm{B}$ sub lattices [10]. 
Table (1): The variation of the Curie-Weiss constant ( $\theta$ ), the effective magnetic moment $\left(\mu_{\mathrm{eff}}\right)$ with Ti-content and field strength.

\begin{tabular}{|c|c|c|c|c|c|c|c|c|c|c|}
\hline & \multicolumn{2}{|c|}{$\mathbf{1 0 1 0}$ Oe } & \multicolumn{2}{c|}{ 13400e } & \multicolumn{2}{c|}{ 16600e } & \multicolumn{2}{c|}{ 19900e } & \multicolumn{2}{c|}{ 23200e } \\
\hline Ti content & $\Theta(\mathrm{K})$ & $\begin{array}{c}\mu_{\text {eff }} \\
(\mathrm{BM})\end{array}$ & $\Theta(\mathrm{K})$ & $\begin{array}{c}\mu_{\text {eff }} \\
(\mathrm{BM})\end{array}$ & $\Theta(\mathrm{K})$ & $\begin{array}{c}\mu_{\text {eff }} \\
(\mathrm{BM})\end{array}$ & $\Theta(\mathrm{K})$ & $\begin{array}{c}\mu_{\text {eff }} \\
(\mathrm{BM})\end{array}$ & $\Theta(\mathrm{K})$ & $\begin{array}{c}\mu_{\text {eff }} \\
(\mathrm{BM})\end{array}$ \\
\hline 0.1 & 811 & 10.7 & 824 & 8.97 & 841 & 7.93 & --- & --- & --- & --- \\
\hline 0.3 & 838 & 8.4 & 855 & 6.41 & 862 & 5.42 & --- & --- & --- & --- \\
\hline 0.5 & 798 & 8.15 & 805 & 5.75 & 817 & 5.16 & --- & --- & --- & --- \\
\hline 0.7 & --- & --- & --- & --- & 790 & 3.03 & 799 & 2.58 & 810 & 2.05 \\
\hline 0.9 & --- & --- & --- & --- & 807 & 3.44 & 814 & 3.03 & 841 & 2.30 \\
\hline
\end{tabular}

The presence of magnetic ordering in the ferrimagnetic region above $\mathrm{x}=0.5$ cannot be explained totally on the basis of the ferrimagnetic to paramagnetic transition but it can be explained on the basis of the temperature evolution of spin-spin correlation within and between A and B sites [21-24].

Fig.(4:a) is a representative curve shows the behavior of the reciprocal magnetic susceptibility versus $\mathrm{T}(\mathrm{K})$ at different field strength of $\mathrm{x}=0.7$ above the Nee'l temperature and these result agree with the previous arguments. The appeared humps in Fig.(4:b) is most likely due to the decrease of the number of aligned dipoles by the magnetic field in its direction to give the increase in $\chi_{M}{ }^{-1}$. In other words the thermal energy exceeds large the magnetic energy, which reflects at a decrease in the magnetic susceptibility (in the paramagnetic region). This can be ascribed also to the increase in the lattice vibration and increasing the disordering in the samples.

Figure (5: a-e) shows the room temperature magnetic hysteresis for the samples $L \underline{i}_{1-x} \underline{T \underline{T}_{x}} \underline{\underline{L} a_{2}} \underline{F e}_{2-y} \underline{O}_{4} \quad 0.1 \leq \mathrm{x} \leq 0.9$ at room temperature. The saturation magnetization $\left(\mathrm{M}_{\mathrm{s}}\right)$, remnant magnetization $\left(\mathrm{M}_{\mathrm{r}}\right)$ and coercivity $\left(\mathrm{H}_{\mathrm{c}}\right)$ follow the same pattern of the lattice parameter as in Table (2). From the data in the figure, the coercivity $\left(\mathrm{H}_{\mathrm{c}}\right)$ increases with increasing Ti content at the expense of $\mathrm{Li}$, with an opposite behavior for $\mathrm{M}_{\mathrm{s}}$ and $\mathrm{M}_{\mathrm{r}}$. This agree well with the relation $\mathrm{H}_{\mathrm{c}} \alpha$ $\mathrm{K}\left(\mu_{0} \mathrm{M}_{\mathrm{s}}\right)^{-1}$ where $\mathrm{K}$ is the anisotropy constant and $\mu_{\mathrm{o}}$ is the permeability of free space[25]. 
(c)

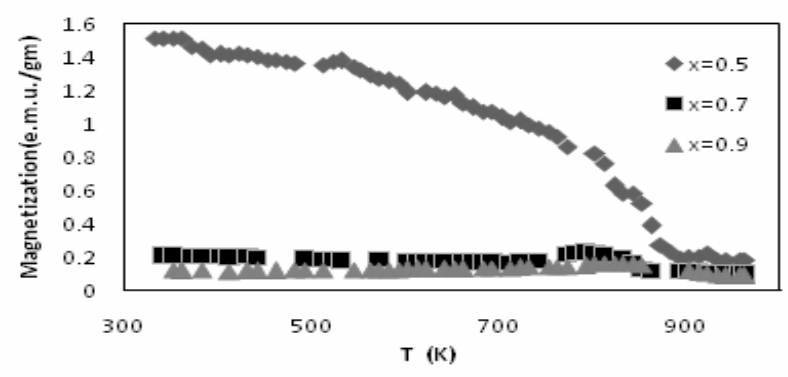

(d)

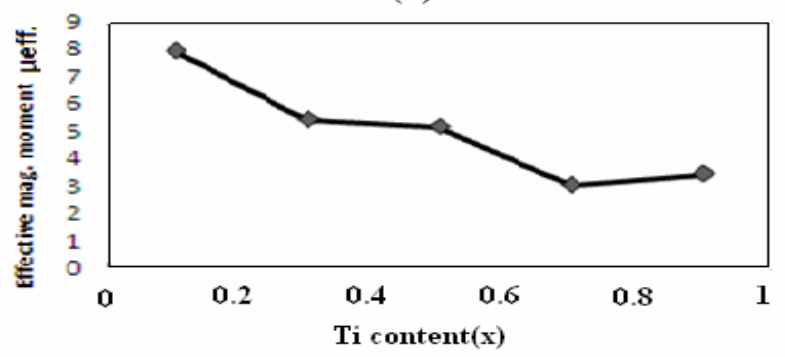

Fig(3: c , d): (c) Variation of magnetization with temperature at different Ti contents, $0.5,0.7$ and 0.9 at field strength 1660 Oe.

(d) Effective magnetic moment vs. Ti content (x)

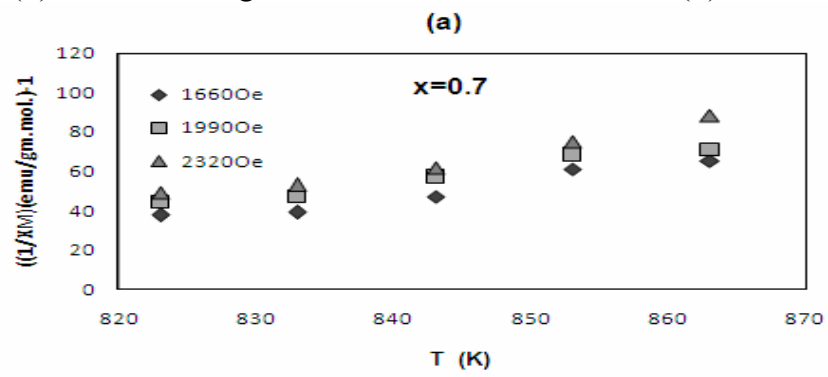

(b)

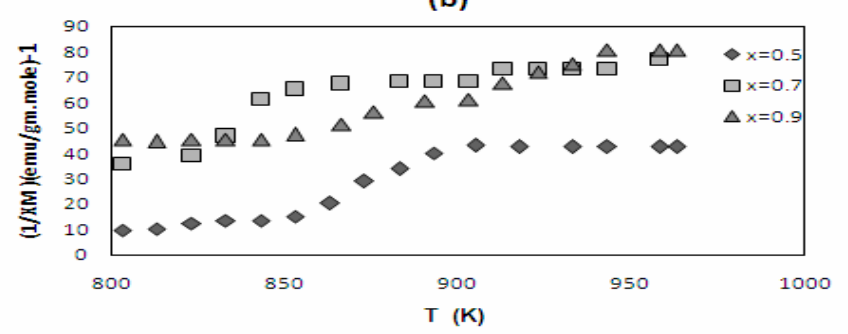

Fig:(4a,b) : Dependence of the reciprocal molar susceptibility on absolute temperature at (a) $x=0.7$ and different magnetic field intensity,

(b) at different $\mathrm{Ti}$ contents, $0.5,0.7,0.9$. 


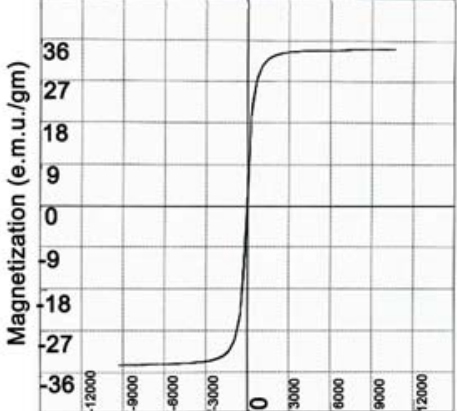

(a) $x=0.1$

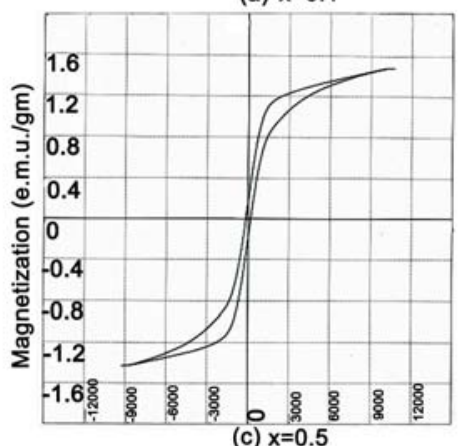

(c) $x=0.5$

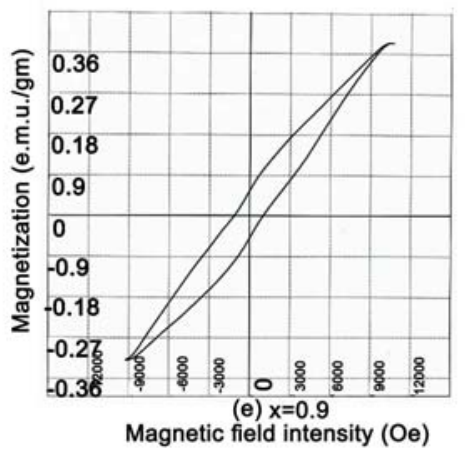

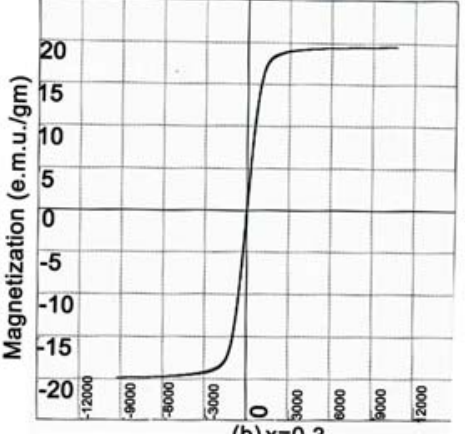

(b) $x=0.3$

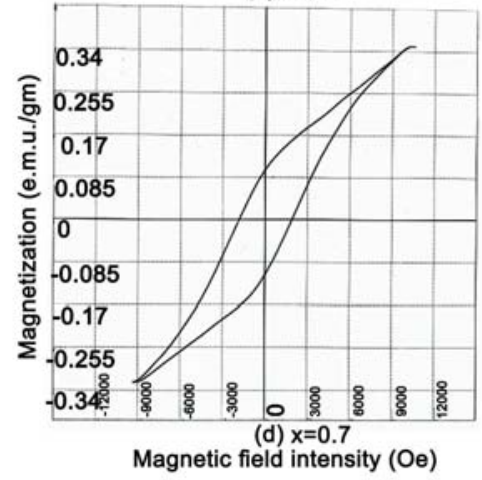

Fig.(5:a-e)Dependence of the magnetization on the magnetic field intensity at room temperature for the samples $\mathrm{Li}_{1-\mathrm{x}} \mathrm{Ti}_{\mathrm{x}} \mathrm{La}_{\mathrm{y}} \mathrm{Fe}_{2-\mathrm{y}} \mathrm{O}_{4 ;} \mathrm{x}=0.1$,

$0.3,0.5,0.7$ and $0.9 ; y=0.1$ 
Table: (2): Values of saturation magnetization $M_{s}$, remanent magnetization $M_{r}$, coercive field $\mathrm{Hc}$ as a function of the Ti content (x).

\begin{tabular}{|c|c|c|c|}
\hline Ti content $(\mathrm{x})$ & $\mathrm{M}_{\mathrm{s}}($ e.m.u. $/ \mathrm{g})$ & $\mathrm{M}_{\mathrm{r}}$ (e.m.u./g) & $\mathrm{H}_{\mathrm{C}}(\mathrm{Oe})$ \\
\hline 0.1 & 34.3 & 4.870 & 24.2 \\
\hline 0.3 & 19.89 & 2.870 & 24.4 \\
\hline 0.5 & 1.482 & 0.272 & 121.9 \\
\hline 0.7 & 0.354 & 0.109 & 1864 \\
\hline 0.9 & 0.372 & 0.053 & 1010 \\
\hline
\end{tabular}

The increase in $\mathrm{H}_{\mathrm{c}}$ with decreasing Li content is mainly due to the variation in the crystal size as the general trend of Fig. (2: b). This can be related to particle size changes according to Kittel's theory [26, 27]. As the particle size depends on the Li content (D) decreases, the coercivity increases and below certain value $\left(D_{s}\right)$, the particles tend to be single domain at which the coercivity has its maximum value. Below $\left(D_{s}\right)$ the coercivity starts to decrease according to the relation $\mathrm{H}_{\mathrm{c}}=(\mathrm{g}-\mathrm{h}) / \mathrm{L}^{3 / 2}$ [28] where, $\mathrm{g}$ is the Lande factor and $\mathrm{h}$ is constant. Below certain value, $\mathrm{H}_{\mathrm{c}}$ becomes very small due to thermal effects which are high enough to demagnetize spontaneously the magnetized particles. The relatively large values of $M_{s}$ as in Table (2) may be due to high degree of crystalinity and uniform morphology of the samples.

The smallest value of $\mathrm{M}_{\mathrm{s}}$ at ( $\mathrm{x}=0.7$ and 0.9$)$ may be due to the mentioned above thermal energy which change the spin structure of the sample from collinear to non-collinear (canted spins). This was enhanced by smaller lattice parameter as shown in Fig. (2: c).

\section{Conclusion:}

The samples $\mathrm{Li}_{1-\mathrm{x}} \mathrm{Ti}_{\mathrm{x}} \mathrm{La}_{\mathrm{y}} \mathrm{Fe}_{2-\mathrm{y}} \mathrm{O}_{4}, 0.1 \leq \mathrm{x} \leq 0.9$ and $\mathrm{y}=0.1$, are prepared and the average lattice parameter and grain size were found to decrease with increasing Ti content on the expense of $\mathrm{Li}$. The data indicated that the decrease in $\mathrm{Tc}$ with increasing $\mathrm{Ti}$ content is due to its masking effect on the ferromagnetic interaction. The magnetic measurements show that the saturation of the magnetization takes place at all field values which are ascribed to alignment of all dipoles in its direction. Also the rearrangement of $\mathrm{Fe}^{2+}$ and $\mathrm{Fe}^{3+}$ between $\mathrm{A}$ and $\mathrm{B}$ sites produce a hump at $\mathrm{x}=0.7$ and 0.9 . The hystresis data showed an increase of $\mathrm{H}_{\mathrm{c}}$ with $\mathrm{x}$ is due to the variation in the crystal size which agrees well with Kittle theory.

\section{Acknowledgement:}


I would like to thank Prof.Dr.M.A.Ahmed for his great effort in helping me with his scientific advices, and using his laboratory. Also, I want to thank him for inspiring and encouraging me to do this work.

\section{References:}

1. W.C.Hsu, S.C.Chem, P.C.Kus, C.T.Lie, W.S.Tsai, Preparation of NiCuZn ferrite nanoparticle from chemical co-percipitation method and the magnetic properties after sintering, Mater. Sci. Eng.; (111):142, (2004).

2. M.C.Rezende, I.M. Martin, R.Faez, M.A.S.Miacci, E.L.Nohara., Ni-Zn nanoferrite for radar-absorbing material, Rev. Fis. Apl.Instr.; 15(12):24, (2002)

3. J. Smit, H.P.J.Wijn, Ferrite, John Wiley and Sons; (1965).

4. D.B.Rogers, R.W.Germann, R.J.Arnott, Crystallographic properties of the spinel systems $\mathrm{Li}_{0.5} \mathrm{Ga}_{2.5-\mathrm{x}} \mathrm{Mn}_{\mathrm{x}} \mathrm{O}_{4}, 0 \leq \mathrm{x} \leq 0.7$, J. Appl. Phys.; 8 (36):2338, (1965).

5. R.He, M.Sun, D.Yu, A study of the Ti-substituted lithium ferrites and its application, IEEE Trans, Magnetics; 6 (18):1622 (1982).

6. M.A.Ahmed, S.T.Bishay, G.Abdelatif, Effect of ytterbium on the electrical properties of Li-Co ferrite, J. Phys. Chem. Solids, (62), 1039 (2001) .

7. M.A.Ahmed, S.T.Bishay, Thermal studies of the electrical conductivity of LiCoYb ferrite. J. Phys. Chem. Solids, 64 (5):769 (2003).

8. E.Schmidbauer, J.Fassbinder, After effect of magnetic susceptibility in FeTi spinels and cation diffusion, J. Magnetism Magnetic Mater, (68), 83 (1987).

9. B.P.Rao, K.H.Rao, Distribution of $\mathrm{In}^{3+}$ ions in indium-substituted Ni-Znferrites, J.Mag.Mag.Mat, 2005; (292):44-48.

10. K.J.Standley, Oxide Magnetic Materials, Clarendon press, Oxford;1972.

11. M.A.Ahmed, N.Okasha, M.A.Gabal, Transport and Magnetic Properties of Co-Zn-La ferrite, Materials Chemistry and Physics, 2004 ; (83):107-113.

12. M.A.Ahmed, S.T.Bishay, The Role of $\mathrm{Dy}^{3+}$ ions and sintering temperature on the Magnetic characterization of Li-Co ferrite, J. Mag. Mag. Mat, (279): 178 (2004).

13. E.Rezlescu, C.Pasnicu, M.L.Craus, P.D.Popa, Effects of rare-earth ions on the quality of Li-Zn ferrite, Cryst. Res. Technol, 3 (31):343 (1996).

14. M.L.Crous, E.Rezlescu, N.Rezlescu, The influence of Fe substitutions by Ge and $\mathrm{Ti}$ in $\mathrm{Ni}_{0.7} \mathrm{Zn}_{0.3} \mathrm{Fe}_{2} \mathrm{O}_{4}$ ferrite, Phys. Stat. Sol. (a), (133), 439 (1992).

15. E.Rezlescu, N.Rezlescu, C.Pasnicu, M.L.Craus, P.D.Popa, The influence of additives on the properties of Ni-Zn ferrite used in magnetic heads, J. Mag. Mag. Mater, (117), 448 (1992).

16. D.Ravinder, Dielectric Behavior of Lithium-Cadmium Ferrites, Phys. stat. sol. (a). (129), 549 (1992). 
17. Xu.Yunhui, S.S.Ata Allah, M.G.Berger, O.Gluck, Rare-earth ion size effect on resistivity, susceptibility, and superconductivity of $\mathrm{RBa}_{2} \mathrm{Cu}_{3-\mathrm{x}} \mathrm{Zn}_{\mathrm{x}} \mathrm{O}_{7-\mathrm{y}}(\mathrm{R}=\mathrm{Yb}, \mathrm{Er}, \mathrm{Y}, \mathrm{Dy}, \mathrm{Gd}, \mathrm{Eu}, \mathrm{Sm}$ and Nd), Phys. rev. B, 53 (22), 15245 (1996).

18. Mamata Maisnam, Sumitra Phanjoubam, H. N. K. Sarma, Chandra Prakash, L. Radhapiyari Devi and O. P. Thakur. Magnetic properties of vanadium-substituted lithium zinc titanium ferrite, Journal of Rare Earths, 25(1): 79 (2007).

19. L.F.Bates, Modern magnetism, Cambridge University press (1961).

20. M.A.Ahmed, E.Atia, L.M.Salah, A.A.El-Gamal, Structural and electrical studies on $\mathrm{La}^{3+}$ substituted Ni-Zn ferrites, Materials Chemistry and Physics, (92), 310 (2005).

21. M.A.Ahmed, N.Okasha, A.Ebrahem. Correlation of the physic chemical properties of Zn-substituted Li-La ferrite, Ceramics International, 31, 361 (2005).

22. B.Viswanathan, V.R.Kmurthy, Ferrite Materials Science and Technology, Narosa Publishing House, (1990).

23. Ibetombi Soibam, Sumitra phanjoubam, C.Prakash. Magnetic and MÖssbauer studies of Ni substituted Li-Zn ferrite, J. Mag. Mag. Mater, 321(18):2779 (2009).

24. K.J.Standly, Oxide Magnetic Materials, Clarendon press. Oxford; (1972).

25. A.G.Bhosale, B.K.Chougule. X-ray, infrared and magnetic studies of Al-substituted Ni ferrites, Mater. Chem. Phys. 97 (2-3), 273 (2006).

26. C.Kittle. Theory of the structure of ferromagnetic domains in films and small particles, Phys. Rev., 70(11-12) : 965 (1946).

27. Kittle C. Domain theory and the dependence of the coercive force of fine ferromagnetic powders on particle size, Phys. Rev, 73(7): 810 (1948).

28. B.D. Cullity, "Introduction to Magnetic Materials", (Addision-Wesley, New York), p 386 (1972). 